\title{
GOOGLE MAPS FOR CROWDSOURCED EMERGENCY ROUTING
}

\author{
Simeon Nedkov ${ }^{\text {a, } * \text {, Sisi Zlatanova }}{ }^{a}$ \\ ${ }^{a}$ GISt, OTB Research Institute for the Built Environment, Delft University of Technology, Delft, The Netherlands - \\ s.b.nedkov@student.tudelft.nl, s.zlatanova@tudelft.nl
}

\section{Commission IV, WG IV/5}

KEY WORDS : Internet/Web, Disaster, Infrastructure, Observations, Mobile

\begin{abstract}
ABS TRACT:
Gathering infrastructure data in emergency situations is challenging. The affected by a disaster areas are often large and the needed observations numerous. Spaceborne remote sensing techniques cover large areas but they are of limited use as their field of view may be blocked by clouds, smoke, buildings, highways, etc. Remote sensing products furthermore require specialists to collect and analy ze the data. This contrasts the nature of the damage detection problem: almost every one is capable of observing whether a street is usable or not. The crowd is fit for solving these challenges as its members are numerous, they are willing to help and are often in the vicinity of the disaster thereby forming a highly dispersed sensor network.

This paper proposes and implements a small WebGIS application for performing shortest path calculations based on crowds ourced information about the infrastructure health. The application is built on top of Google Maps and uses its routing service to calculate the shortest distance between two locations. Impassable areas are indicated on a map by people performing in-situ observations on a mobile device, and by users on a desktop machine who consult a multitude of information sources.
\end{abstract}

\section{INTRODUCTION}

Effective management of a disaster depends on knowledge about the health and condition of the infrastructure. An important analysis in the response phase of a disaster management operation is the calculation of shortest routes between two locations. Routing, although a simple task on itself, becomes difficult during disaster relief operations where a lack of information is the norm. Collecting information about the state of the infrastructure is a complex task due to the extent of the area and quantity of needed observations. Fortunately, making these observations does not require much specialization as almost everyone can judge whether a street is passable. At the same time numerous people on the ground are not able to directly aid the relief operation due to lack of equipment or knowledge, but nonetheless are willing to help. It comes therefore to mind to leverage the knowledge and large numbers of the crowd in collecting information about the state of the infrastructure and more.

Crowdsourcing and web mapping are becoming increasingly common. Crowdsourcing has shown its strengths in endeavours such as Wikipedia. Web mapping platforms such as Google Maps and Bing Maps have revolutionized cartography and have brought it to the masses. New web technologies have made creating dynamic and intelligent websites easier. The combining of crowdsourcing and web mapping technologies have produced OpenStreetMap. The field of Disaster Management has also benefited from this combination in the form of Ushahidi, a mapping web application used in disaster relief operations to map hazards and people's needs in disaster stricken areas. Using Ushahidi the "crowd" is able to collect, store and share information about events and points of interest in the disaster area. They can identify blocked roads, shelter locations, people in need of immediate medical attention, etc. Rescue workers use this information to quickly get an overview of the needed aid and plan their operations around that information. However, Ushahidi does not support geospatial analyses. More specifically, it is not possible to perform shortest route calculations.

This paper presents our investigations on crowdsourcing for disaster management purposes. What is crowdsourcing? What constitutes crowdsourcing emergence? The found constituents are implemented in a small WebGIS application that uses the "knowledge of the crowd" to aid the routing challenges rescue workers face during the response and recovery phases of disaster management. The goal of proposed application is twofold: 1) automate the way finding process in a disaster stricken area by 2) enabling volunteers to act as sensors and report on the infrastructure health.

The paper is organized as follows: section 2 discusses what crowdsourcing is and what stimulates its emergence and growth. The section continues by discussing what a WebGIS is and what functionality is expected from it. Section Error! Reference source not found. outlines the used technologies. Section Error! Reference source not found. show highligths the methodology and obtained results. Section 5 presents some conclusions while section 6 identifies possible research directions.

\section{CROWDSOURCED DIS AS TER MANAGEMENT}

The sudden change of urban infrastructure configuration and health immediately after a disastrous event renders much of

\footnotetext{
* Corresponding author.
} 
existing urban infrastructure information useless and out-dated. At the same time, information about infrastructure health is vital as a large part of rescue operations make use of roads, bridges and tunnels. Finding the shortest route in a city stricken by a natural disaster is a complicated operation that is no longer a function of distance and travel time only, but also of the infrastructure state. Obtaining up-to-date infrastructure information quickly is important, but also very challenging due the large extent of urban areas and the lack manpower.

Airborne and satelliteborne observation techniques seem to offer a solution but challenges lurk there too. Although the coverage of remote sensing images is sufficient, it is not always possible to make observations due to clouds, bad weather or simply because some damages can only be observed and assessed from the ground. Turning this data into useful information requires a considerable amount of time and effort from specialized personnel. What is thus needed is a complementary sensor network of some kind that is capable of performing infrastructure health measurements that can be used to aid the routing process.

Two things suggest outsourcing data collection to the crowd: 1 ) the task at hand is not overly complex and 2) a large group of people is willing and able to help. Goodchild (2007) recognizes the crowd's potential by highlighting that each individual is in essence a sensor, while the crowd as a whole forms a sensor network. Laituri and Kodrich (2008), and Shirky (2009) show that the crowd is capable of more than data collection only. Citizens and other non-specialists can greatly support collection of ground information if they are provided with appropriate tools. Heipke (2010) furthermore notes that data collected by local mappers and observers tends to be of higher quality than information gathered by someone from abroad who is not familiar with the environment.

\subsection{Internet for ODRC}

Disaster management is seen to be the task of official organizations such as governments and humanitarian nongovernmental none-profit organizations. Until now, these institutions have largely remained off the internet. Laituri and Kodrich (2008) identify a move towards usage of internet technologies in the form of online disaster-response communities (ODRC). They identify three tiers. The first tier consists of a network of traditional national and international organisations that are responsible for raising awareness and financial funds prior to the occurrence of a disaster.

The second tier holds the groups and organizations that respond immediately after a disaster strikes. This tier is largely filled by organizations from the first tier. Instead of raising awareness, they are now coordinating action. Since recent years, the second tier is expanding to house informal organizations and networks that wish to contribute to disaster management and rescue operations. Their activities involve using internet and social media to share information about the disasters in the form of pictures, blogs, videos, wiki's and links to official sources of information.

The third tier is using the internet technology for more than information distribution only. This tier consists of technology savvy volunteers who are able to collect geographical information and/or build geospatial analysis tools that aid during, but also after the disaster management process. Examples of these endeavours are the OpenStreetMap mappers, Ushahidi creators and users, and communities such as Crisis Mappers ${ }^{1}$ and CrisisCommons ${ }^{2}$. Third tier contributions and tools are not limited to geographical information and "traditional" sensing and observation techniques, but include new and cutting edge technologies. For instance, the 2011 Japan earthquake and nuclear power plant failure have moved people to install Geiger counters and stream the measurements to the world through Pachube ${ }^{3}$. OpenStreetMap can be put in the same light as being more than an aggregation of GPS tracks. Some parts are synthesized from satellite images by the process of digitization i.e. people tracing roads and rivers over satellite images. The "crowd" is thus capable of more than data collection only, it is able and willing to perform more complex task such as mapping, geographical analy sis and programming.

\subsection{Crowdsourcing}

Crowdsourcing is the coming together of a diverse (in terms of knowledge, background, specialisation and interests) group of people who, using modern internet technology, perform complex tasks that normally are performed by specialists and professionals (Goodchild 2007, Goodchild 2010, Laituri and Kodrich 2008). Crowdsourcing has become possible due to recent advances in internet technology and specifically due to advances in communication technologies (Shirky 2009). Social media such as blogs, wiki's, Twitter and Facebook have increased ease of communication by providing more and better streamlined communication channels thereby reducing the transaction cost (in terms of spent time and money) of communicating with other people (Shirky 2009). This decrease in communication transaction cost results in the emergence of highly dispersed but effective loosely organized groups of people who share a common interest and work towards a common goal. Social media technologies allow groups to selforganize and manage, thereby removing the need for a managerial layer that is inherent to large organizations. Such a group becomes cheaper, thereby allowing them to undertake tasks previously deemed too expensive in terms of time, money and manpower. The loose organization keeps the group flexible and agile, allowing them to quickly adapt to changing situations. The reduction in transaction cost enables "every one to communicate with everyone" thereby exposing the product under development to many eyes and as many disciplines and expertise. The total amount of knowledge and expertise increases which in turn allows the completion of complex tasks

\footnotetext{
${ }^{1}$ www.crisismappers.net

${ }^{2}$ www.crisiscommons.org

${ }^{3}$ www.pachube.com
} 
(Shirky 2009, Laituri and Kodrich 2008). Crowdsourcing thus thrives only when sufficient communication channels are available. Applications aiming to leverage crowdsourcing must supply the crowd with sufficient and efficient means of communication.

Crowdsourcing strengths and weaknesses: crowdsourcing is slowly finding its way into disaster management (Lucaszczyk 2011). Amongst the numerous crowdsourcing virtues, the following are recognised to be valuable for disaster management.

Speed: crowdsourcing initiatives need little effort to materialize and start cooperating. An Ushahidi instance can be up and running in two hours. The OpenStreet mappers have produced completely new and highly detailed maps of Haiti in the course of days (Harvard Humanitarian Effort 2011). Traditional organizations tend to be slower in their response (Shirky 2009). Up-to-date data: crowdsourced data can be collected at a tremendous pace and kept fresh due to the "many eyes watching" principle. Information is shared easily through Ushahidi and Shahana, but also through blogs, Twitter, Facebook, etc. while geographical information can be distributed through platforms such as GeoNode and other OGC products.

Wide knowledge pool: as discussed above, crowdsourcing initiatives are characterized by a widely diverse group of contributing volunteers.

Momentum: Due to their openness (crowdsourcing initiatives use the web to communicate and open source tools to collaborate) crowdsourcing initiatives gain momentum faster and keep it going for longer than closed organizations.

Continuity: a substantial part of volunteered (geographical) information or disaster management software is the product of free time activity and, to a lesser degree as a by-product of commercial processes. As such, volunteers are constantly working on, and are surrounded by the information and tools that they later deploy and use during a disaster management operation. The so created continuity ensures an efficient and effective deployment and usage of the technologies. Although official disaster management agencies organize training sessions, disaster management is often one of their many tasks and is certainly not a day-to-day experience.

The biggest threat to acceptance of crowdsourcing results, and especially data, has always been the question of reliability and robustness (accuracy when talking about data). Flanagin and Metzger (2008) discuss these issues in terms of believability or credibility-as-perception. The degree of believability is determined by trustworthiness and expertise. Goodchild (2007) and Schmitz et al. (2008) note that volunteered efforts can be trustworthy even when not produced by experts by relying on the collective "wisdom" of the crowd to detect and correct inaccurate information entries, keep the data set up-to-date and "defend" it from vandals and bugs.

WebGIS for crowdsourced disaster management: Traditional GI systems are holistic, heavy weight solutions i.e. a single GI system is designed to solve many a problem. GI systems need powerful desktop computers, constraining GIS experts to a desk. GIS 'in the field' e.g. in the hands of first responders and volunteers has not seen a lot of practical application.

The dynamic nature of modern web pages and applications, made possible by Web 2.0 technologies has started to move GIS away from desktop machines. These technologies make it easy to connect mobile applications to GIS servers through the Internet in an interactive manner. Mobile devices have become gateways to powerful servers that house geographical data and perform complex analyses. Such mobile and lightweight GI systems are called WebGIS. From the user's point of view, a WebGIS is capable of performing the standard GIS operations, but now users can take that functionality with them on the road. Although several web based emergency management systems are available, the spatial analyses supported by them are limited. Acuna et al. (2010) have surveyed these systems and based on their findings and performed literature studies, have identified several design patterns they deem necessary for disaster management web applications. These are listed as

- $\quad$ Awareness for First Responders: fast and dynamic access to information regarding the emergency at hand.

- Collective Memory / (Temporal) Data Archives

- Tabular Information Presentation

- Map-based Navigation and Information presentation

- Data authoring: mechanisms for attaching author and source information to data items.

- Display of up-to-date Data

- Mechanism for Direct Data Manipulation

- $\quad$ Style Sheets for Multiple Media Types

- Hand-held Devices

Neis et al. (2010) present an emergency routing application based on OpenStreetMap data and Open Location Services Route Service. Their application supports shortest path analysis on a damaged infrastructure network by allowing users to mark blocked roads by drawing lines. The application is built inside Ushahidi and thus leverages its crowdsourcing powers. Drawbacks of this implementation are its interface, speed and a lack of mobile client.

\section{TECHNOLOGY}

Based on the crowdsourcing and disaster management design patterns discussed in sections 2.1 and 2.2, a small WebGIS has been implemented that aims to enhance and automate the disaster management activity of route finding with information gathered through crowdsourcing.

The built application is a client-server configuration. The client side runs on HTML/JavaScript and communicates through asyncrhonous Javascript and XML or AJAX with a RESTful server. The JavaScript library jQuery ${ }^{4}$ is used to implement AJAX. REST is an abbreviation of REpresentational State Transfer, a "software architecture for distributed hypermedia systems such as the World Wide Web" (Pautasso et al. 2008). In this architecture, every resource is stored on a server and has a

\footnotetext{
${ }^{4}$ http://www.jquery.com
} 
unique identifier. In web-based systems, this identifier is known as a Unified Resource Identifier (URI). Clients access and manipulate the resources by standard HTTP methods. Obtaining the current state of a resource, for instance, is done by sending a HTTP GET request to a resource's URI. The server processes the request and sends a representation of the resource's current state as a XML, JSON (the lightweight datainterchange format JavaScript Object Notation) or simply as a plain text document.

REST is implemented using Django ${ }^{5}$. Django is a Python web application framework that eases web application development by automating and abstracting low-level tasks and operations and by automatically building the database schema. Developers can then focus on the application logic. The GeoDjango plugin makes Django spatially enabled. GeoDjango uses open source libraries such as GDAL/OGR and GEOS to interact with and manipulate geographical information. Django is chosen for its simplicity, ease of deployment and very high quality documentation. Alternatives such as GeoServer are full-fledged applications that offer more functionality than is currently required.

Data is stored in two databases: PostGIS and Google Fusion Tables. PostGIS is chosen as the main data store as it is a wellknown, powerful, open source spatial database. It enjoys the momentum discussed in section 2.2. Google Fusion Tables ${ }^{6}$ is "a cloud-based data management and integration service" that is designed specifically with collaboration, data sharing, the Web and usage by non-technical users in mind (Gonzalez et al. 2010b). Fusion Tables stores (geographical) data in tabular form. Tables can be shared with the world or a selected group of people all of which can have different roles i.e. viewers and editors. The data is accessible through a Web interface and can be visualized in several different ways including as geometry on a map. Tables storing geographical information can be exported as KML.

The used mapping framework is Google Maps. The WebGIS application is hosted on the author's website ${ }^{7}$. The source code is hosted on the code collaboration website Github ${ }^{8}$.

\section{METHODOLOGY AND IMPLEMENTATION}

Geographical Information Systems have four main capabilities: data gathering, data storage and management, data analy sis and presentation. The application presented in this paper extends these by implementing the design patterns and crowdsourcing mechanism described earlier on in order to create a WebGIS fit for disaster management.

Non-expert users interact with the application through a desktop interface and a mobile interface. To identify any

\footnotetext{
${ }^{5}$ https://www.djangoproject.com/

${ }^{6} \mathrm{www}^{\text {google.com/fusiontables }}$

${ }^{7}$ http://gmer.ndkv.nl/

${ }^{8} \mathrm{http}: / /$ www.github.com/ndkv/gmer/
}

obstacle, users draw a polygon on the map or put a simple marker at the location of the blockage. Desktop users asses the condition of the infrastructure by tapping into geographical information sources such as satellite images, official reports, etc., but also into social media data sources such as blogs, Facebook, Twitter, etc. The mobile interface allows for the collection of data through a mobile device such as a smartphone. Mobile users act as sensors and report on the status and health of the infrastructure. Expert users interact with the application by way of an API.

The following sections cover the four GIS components and discuss how these have been extended, what user interactions take place and which disaster management and crowdsourcing design patterns are implemented.

Data gathering, storage and management: The two types of interfaces have similar functionality: users are able to mark blocked streets and areas, create routes, read obstacle metadata and read and write comments about individual obstacles. Both interfaces display the same information i.e. the desktop users see what the mobile users have mapped and vice versa.

The desktop interface has four panels: a map, obstacle and route creation controls, obstacle properties panel and a comments panel (Figure 1). Users create obstacles and routes using the controls under the map. An obstacle is defined by drawing a polygon on the map that represents a blockage. Users define routes by providing a point of departure and a point of arrival after which the implemented routing algorithm finds the shortest path around all obstacles. See Nedkov and Zlatanova (2011) for a detailed discussion on the routing algorithm.

The panels to the right of the map harbour the crowdsourcing mechanisms discussed in section 2.2. The properties panel provides an overview of obstacle metadata (e.g. id, owner, creation date, type, etc.), and controls to modify obstacle geometry and delete obstacles. The comments tab acts as the main communication channel. Users can discuss the each obstacle separately. As such, discussion can be held on the origin, validity, history, etc. of each obstacle.

The mobile interface looks simpler than its desktop counterpart but contains almost the same functionality. Users select polygons by tapping on them. Obstacles are created by entering points instead of polygons since we believe that drawing polygons may prove too cumbersome on a touch screen device. However, the routing algorithm needs polygons to operate properly so the points must be turned into polygons. This is done by the desktop users who look at the points and connect them in order to create polygons. The points are removed once the polygon is drawn.

PostGIS acts as the main data store while Fusion Tables acts as a non-expert data dissemination and backup, and cooperation facility. PostGIS stores obstacle geometries which are versioned i.e. modifying obstacle geometry does not overwrite the original 
geometry, but creates a new version of it. All versions are kept indefinitely thereby adding a temporal dimension to the system.

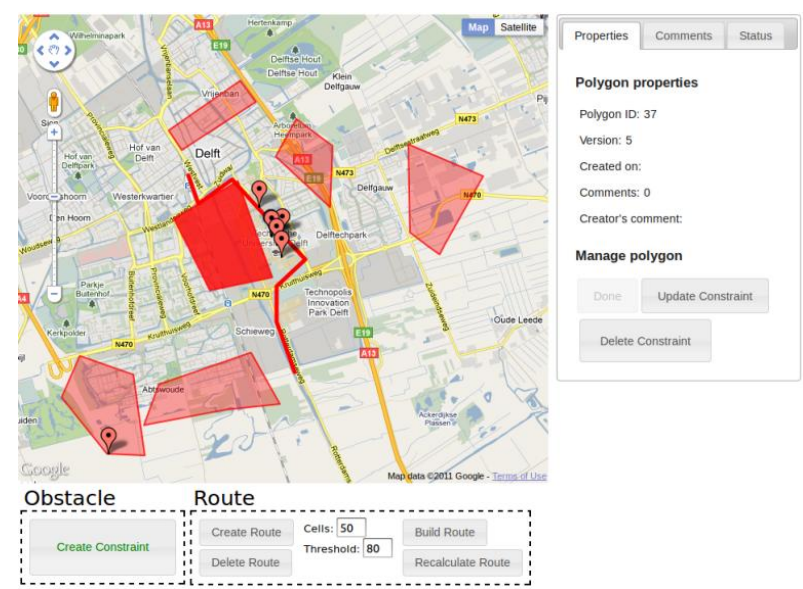

Figure 1. The desktop interface. Polygons denote real-world obstacles.

Figure 2 shows an overview of the system's components. Users' observations are saved in the PostGIS database first and move later to GFT. GFT holds the latest snapshot of the data, whereas PostGIS stores all data ever entered.

Analysis and cooperation: One of the presented application's main merits is the stimulation of crowdsourced data collection. As already noted, the crowd is capable of more than data collection only. To leverage this capability, the built application supports the calculation of the shortest route. The routing functionality turns the application into more than a data silo. The purpose of this analysis is twofold. On one hand it provides rescue workers with an automated shortest path analy sis tool. On the other hand it acts as a return of investment for the crowd as they can use their own data for their own routing needs. The people's usage of their own data acts as an incentive to generate better and accurate data, and keep it up to date.

Users are encouraged to cooperate on several different levels. First, they can work together on collecting the most accurate information they can find through all the means they are comfortable with. For instance, some may draw information from their Twitter network, whereas others may have some experience working with satellite images. Both types of users can enter their data in the application and compare the results. A third user may then check both of their results. Another type of cooperation is found between the desktop and mobile users. The mobile users' task is to make quick and numerous observations which the desktop user then synthesizes into polygons. Both users benefit: the mobile user can work autonomously without worrying about what other mobile users are doing, while the desktop user can observe the whole operation from a higher vantage point using more powerful hardware without needing to worry about the difficulties of working in the field.

The tools available for collaboration are the comments section on the desktop and mobile interfaces and the communication facilities of GFT. GFT allows users to discuss all facets of the data in its tabular view. Users can place comments on columns, rows and individual cells.

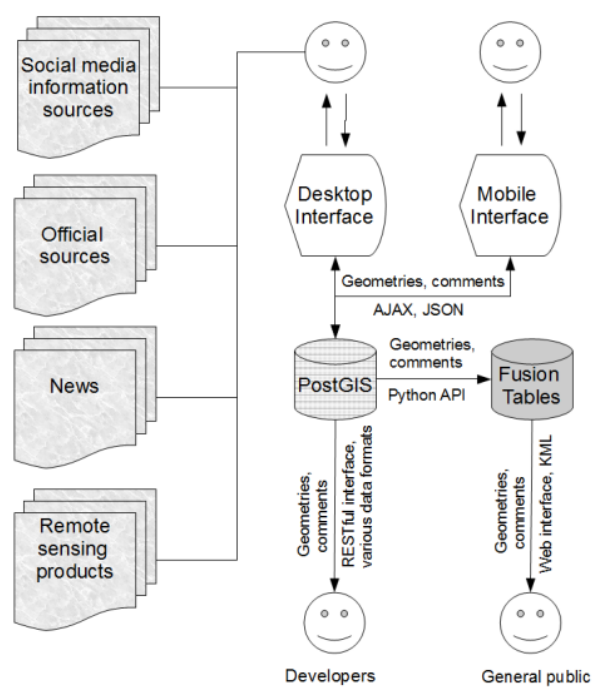

Figure 2. The system's components. Data streams are represented by arrows. The users on top are the data collectors. They gather data and enter it through the desktop and mobile interfaces. The text on top and left of the lines denote the payload of the data stream while the text on the bottom and right of the lines denotes the used technology

Data presentation, visualisation and sharing: as discussed in section 2.2, crowdsourcing initiatives are successful and gain momentum when they are open and tuned to the user's capabilities and needs. In terms of storage, open means that the data is easy to access. Two user groups are targeted here: "normal" users (informal part of second tier) who are not able or willing to work with raw data and prefer a pre-processed version of it, and savvy computer users (volunteers in the third tier) who want access to the raw data. The built application satisfies the needs for these two groups through the following means: the web and mobile interfaces, GFT's export functionalities and visualisations, and the developer API. The web and mobile interfaces allow people to not only input data, but to also browse the collected information. GFT's webinterface supports tabular and mapped visualisations. The data can be extracted as KML. The API gives access to the data in developer friendly formats such as Well-known Text, KML, GeoJSON, etc.

\section{CONCLUSION}

Crowdsourcing is the unplanned coming together of a highly diverse group of people who use the latest technology and data formats and sources available to aid a certain disaster management cause. It is difficult to predict beforehand how many people with what skills will participate and which tools they will deploy. Designing an application for a crowdsourcing effort therefore seems counter to its volatile nature. What is needed, rather, is not a complete system, but a set of components which are well developed, well documented and can 
seamlessly be integrated on the fly to provide for the situation's needs. This will allow systems to be configured with respect to disaster management needs.

The main strength of the built WebGIS compared to other web disaster management solutions is its ability to perform spatial analy sis in the form of routing. The users are not only gathering data for others e.g. relief organizations, but also for themselves as they too can use the routing service. The quality of provided information is expected to rise once data gatherers experience first-hand how the provided data is used and how errors affect the routing solution. Data providers and gatherers' mindset might change from "contribute occasionally and forget" to "contribute continuously and guard quality". The implemented communication methods aim at creating a long lasting community.

Parallels between upcoming crowdsourced disaster management initiatives and the open source communities are important to notice and foster: computer savvy users fiddle daily with the technology they later use for disaster management. As such, they have momentum and a running, hands-on experience with used technologies. Using open source is vital as it enables hackers to adapt the software to their needs on the fly. Open sourcing a project allows more people to get involved, which in turn results in a larger knowledge and contributing user base.

\section{FUTURE WORK}

The application's fitness for use has to be evaluated by deploying it in a real-world simulation. The implemented ideas and principles are based on theory and have been tested in a research environment.

Trust is an important commodity in crowdsourced projects. Mechanisms for increasing trust and checking the trustworthiness of data sources should be researched and implemented. Several ways of trust generation have been brought forward, one of which is communication. For the current application, an extra communication channel is for instance an application-wide chat module. Users will then be able to discuss all aspects of the application, not only the obstacles. Implementing a user management system further strengthens the trust validation process by allowing the examination of the contributors' past actions.

An in-depth study of obstacle input methods has to be performed. Drawing polygons may not be the most intuitive input method available. Research and field trials may be needed to assess the best input methods.

Currently, an internet connection is required for the prototype to function. The availability of working wireless networks cannot be taken for granted during disasters. Therefore a caching mechanism needs to be implemented that enables the prototype to function in the absence of internet connectivity.

Google Maps is chosen due to a lack of open source solutions at the time of the here presented application's inception. The situation has changed considerably. The OpenStreetMap mapping success in Haiti suggests using OpenStreetMap data in combination with OpenLayers.

\section{REFERENCES}

Acuna, P., Diaz, P., \& Aedo, I. (2010). Development of a design patterns catalog for web-based emergency management systems. In Proceedings of the 7 th International ISCRAM Conference. ISCRAM

Botterell, A., \& Griss, M. (2011). Towards the next generation of emergency operation systems. In Proceedings of the 8 th International ISCRAM Conference. ISCRAM.

Douglas, D.H., \& Peucker, T.K. (1973). Algorithms for the reduction of the number of points required to represent a digitized line or its caricature. Cartographica: The International Journal for Geographic Information and Geovisualization, 10(2), 112-122.

Flanagin, A., \& Metzger, M. (2008). The credibility of volunteered geographic information. GeoJournal, 72(3), 137-148.

Francia, S. (2011). Soap vs. rest. http: / / spf13.com/post/soap-vs-rest

Gonzalez, H., Halevy, A.Y., Jensen, C.S., Langen, A., Madhavan, J., Shapley, R., Shen, W., \& Kidon, J.G. (2010b). Google fusion tables: web-centered data management and collaboration. In Proceedings of the 2010 international conference on Management of data, SIGMOD '10, (pp. 1061-1066). New York, NY, USA: ACM.

Goodchild, M. (2007). Citizens as sensors: the world of volunteered geography. GeoJournal, 69(4), 211-221.

Goodchild, M.F., \& Glennon, J.A. (2010). Crowdsourcing geographic information for disaster response: a research frontier. International Journal of Digital Earth, 3(3), 231-241.

Harvard Humanitarian Effort (2011). Disaster relief 2.0 report The future of information sharing in humanitarian emergencies Washington, DC and Berkshire, UK http://bit.ly/p0wSW3

Heipke, C. (2010). Crowdsourcing geospatial data. ISPRS Journal of Photogrammetry and Remote Sensing Volume 65, Issue 6, November 2010, Pages 550-557

Laituri, M., \& Kodrich, K. (2008). On line disaster response community: People as sensors of high magnitude disasters using internet GIS. Sensors, 8(5), 3037-3055.

Lukaszczyk, A. (2011). International experts blend space technologies and crowdsourcing to enhance disaster management tools.

http://www.newswise.com/articles/international-experts-blendspace-technologies-and-crowdsourcing-to-enhance-disastermanagement-tools

Nedkov S., S. Zlatanova (2011). Enabling obstalce avoidance for Google Maps' navigation service. In: O. Altan, R. Backhous, P Boccardo, D. Stevens, S. Zlatanova (Eds.); Proceedings Gi4DM 2011, Antalya, 6 p.

Pautasso, C., Zimmermann, O., \& Leymann, F. (2008). Restful web services vs. "big"' web services: making the right architectural decision. In Proceeding of the 17th international conference on World Wide Web, WWW '08, (pp. 805-814). New York, NY, USA: ACM.

Pucher, A. (2009). Usability and implementation issues for cartographic information systems in interdisciplinary environments.

Neis, P., Singler, P., Zipf, A. (2010). Collaborative mapping and Emergency Routing for Disaster Logistics - Case studies from the Haiti earthquake and the UN portal for Afrika. In Proceedings of the Geoinformatics Forum Salzburg.

Shirky, C. (2009). Here comes everybody : the power of organizing without organizations. Penguin.

Tornqvist, E., Sigholm, J., \& Nadjm-Tehrani, S. (2009). Hastily formed networks for disaster response: Technical heterogeneity and virtual pockets of local order. In Proceedings of the 6th International ISCRAM Conference. ISCRAM. 